\title{
BMJ
}

\section{Effect of interpregnancy interval on outcomes of pregnancy after miscarriage: retrospective analysis of hospital episode statistics in Scotland}

\author{
Eleanor R Love, medical student, ${ }^{1}$ Siladitya Bhattacharya, professor of reproductive medicine, ${ }^{1}$ Norman C \\ Smith, consultant obstetrician, NHS Grampian, ${ }^{2}$ Sohinee Bhattacharya, lecturer in obstetric epidemiology ${ }^{3}$
}

$\overline{1}$ University of Aberdeen, Aberdeen

${ }^{2}$ Department of Obstetrics and Gynaecology, Aberdeen Maternity Hospital, Aberdeen

${ }^{3}$ Dugald Baird Centre for Research on Women's Health, Aberdeen Maternity Hospital, Aberdeen

AB25 2ZD

Correspondence to: Sohinee

Bhattacharya

sohinee.bhattacharya@abdn.ac.uk

Cite this as: BMJ 2010;341:C3967 doi:10.1136/bmj.c3967

\section{ABSTRACT}

Objective To determine the optimum interpregnancy interval after miscarriage in a first recorded pregnancy. Design Population based retrospective cohort study. Setting Scottish hospitals between 1981 and 2000. Participants 30937 women who had a miscarriage in their first recorded pregnancy and subsequently became pregnant.

Main outcome measures The primary end point was miscarriage, live birth, termination, stillbirth, or ectopic pregnancy in the second pregnancy. Secondary outcomes were rates of caesarean section and preterm delivery, low birthweight infants, pre-eclampsia, placenta praevia, placental abruption, and induced labour in the second pregnancy.

Results Compared with women with an interpregnancy interval of 6-12 months, those who conceived again within six months were less likely to have another miscarriage (adjusted odds ratio $0.66,95 \%$ confidence interval 0.57 to 0.77$)$, termination $(0.43,0.33$ to 0.57$)$, or ectopic pregnancy $(0.48,0.34$ to 0.69$)$. Women with an interpregnancy interval of more than 24 months were more likely to have an ectopic second pregnancy (1.97, 1.42 to 2.72$)$ or termination $(2.40,1.91$ to 3.01$)$. Compared with women with an interpregnancy interval of 6-12 months, women who conceived again within six months and went on to have a live birth in the second pregnancy were less likely to have a caesarean section $(0.90,0.83$ to 0.98$)$, preterm delivery $(0.89,0.81$ to 0.98$)$, or infant of low birth weight $(0.84,0.71$ to 0.89$)$ but were more likely to have an induced labour (1.08, 1.02 to 1.23$)$. Conclusions Women who conceive within six months of an initial miscarriage have the best reproductive outcomes and lowest complication rates in a subsequent pregnancy.

\section{INTRODUCTION}

Miscarriage or spontaneous pregnancy loss before 24 completed weeks of gestation affects one in five pregnancies. ${ }^{1}$ Women with an initial miscarriage are at increased risk not only of a second miscarriage ${ }^{2}$ but also of complications in a subsequent pregnancy. Compared with women who have never been pregnant, those who have miscarried are more likely to experience threatened miscarriage, preterm birth, induced labour, and postpartum haemorrhage in the next pregnancy. ${ }^{3}$

Any miscarriage is associated with distress and prompts questions about the optimal timing of the next pregnancy. How long a couple should wait before trying for another pregnancy after a miscarriage is controversial. Some clinicians believe that there is little justification for delaying the next pregnancy, ${ }^{4-6}$ as an increased interpregnancy interval is unlikely to improve perinatal outcomes, ${ }^{2}$ whereas a new viable pregnancy and the birth of a child could enhance the women's chances of recovery. ${ }^{48}$ Others suggest waiting at least six months for full mental and physical recovery before another pregnancy. Current guidelines from the World Health Organization recommend that women should wait for at least six months before trying again, ${ }^{9}$ whereas others suggest a delay of up to 18 months, based on reports that interpregnancy intervals of 18-23 months after a live birth can enhance maternal and perinatal outcomes in the next pregnancy. ${ }^{10-13}$ This length of delay, however, poses a particular problem for women in the Western world as they tend to delay childbearing for several social and economic reasons. Women over 35 are more likely to experience difficulties in conceiving and women aged 40 have a $30 \%$ chance of miscarriage, which rises to $50 \%$ in those aged 45 or more. ${ }^{14}$ Any delay in attempting conception could further decrease the chances of a healthy baby. Many women are aware of this potential problem, which is why first time mothers aged 35 or over are more likely to plan a second pregnancy shortly after their first. ${ }^{15}$

We compared maternal and perinatal outcomes in women with different interpregnancy intervals after a miscarriage in an initial pregnancy. The Scottish morbidity records collect information on all hospital admissions in Scotland and therefore offer an ideal opportunity to examine the effects of interpregnancy intervals after miscarriage on outcomes of the subsequent pregnancy. 


\begin{tabular}{|c|c|c|c|c|c|c|}
\hline \multirow[b]{2}{*}{ Characteristics } & \multicolumn{5}{|c|}{ Interpregnancy intervals (months) } & \multirow[b]{2}{*}{$P$ value } \\
\hline & $<6(n=12744)$ & $6-12(n=7791)$ & $12-18(n=2958)$ & $18-24(n=1995)$ & $224(n=5449)$ & \\
\hline \multicolumn{7}{|c|}{ Mean (SD) age during pregnancy: } \\
\hline First pregnancy & $26.0(5.4)$ & $25.9(5.8)$ & $25.6(6.0)$ & $25.3(6.2)$ & $23.9(5.9)$ & $<0.01$ \\
\hline Second pregnancy & $26.9(5.4)$ & $27.3(5.8)$ & $27.4(6.0)$ & $27.6(6.2)$ & $28.7(5.9)$ & $<0.01$ \\
\hline \multicolumn{7}{|l|}{ Carstairs category 1-4: } \\
\hline First pregnancy & 4449 (34.9) & 2621 (33.6) & $916(31.0)$ & $623(31.2)$ & $1547(28.4)$ & $<0.01$ \\
\hline Second pregnancy & $4724(37.1)$ & $2773(35.6)$ & $1001(33.8)$ & 677 (33.9) & $1763(32.4)$ & $<0.01$ \\
\hline \multicolumn{7}{|l|}{ Smoking status: } \\
\hline Ever smoked & $1261(9.9)$ & $835(10.7)$ & $398(13.5)$ & $290(14.5)$ & $963(17.7)$ & \multirow{3}{*}{$<0.01$} \\
\hline Never smoked & $4230(33.2)$ & $2308(29.6)$ & $845(28.6)$ & $575(28.8)$ & $1543(28.3)$ & \\
\hline Not known & $7253(56.9)$ & $4648(59.7)$ & $1715(58.0)$ & $1130(56.6)$ & $2943(54.0)$ & \\
\hline
\end{tabular}

\section{METHODS}

The Information Services Division of the National Health Service, Scotland, is a national organisation for health information, statistics, and computing services and has been in existence for over 40 years. Anonymised patient based data for inpatient and day case activity in the specialty of obstetrics are routinely collected from the whole of Scotland as Scottish morbidity records. We used information from this database (specifically SMR01 and SMR02) for this study. The database is subjected to regular quality assurance checks and has been more than $99 \%$ complete since the late 1970 s. $^{16}$

We used a retrospective cohort study design where exposure was taken as increasing interpregnancy intervals between a first and second pregnancy. We extracted data on all women who had a miscarriage recorded for a first pregnancy between 1981 and 2000 in Scotland and went on to have a subsequent pregnancy, as identified by internal linkage of SMR01 and SMR02 datasets using probability matching. Women who had twin or multiple pregnancies were excluded.

To determine the interpregnancy interval we calculated the time interval between the pregnancy related admission to hospital, and then subtracted the number of weeks' gestation of the second pregnancy at the time of the second admission, as recorded in the database. We then divided the women into five groups depending on the time interval between the two pregnancies: less than six months, $6-12$ months, $12-18$ months, 18-24 months, and more than 24 months. Our reference category was an interpregnancy interval of 6-12 months.

We assumed admission dates less than four weeks apart for first and second pregnancy events to be related to the same event, and we excluded the relevant woman from analysis. We also excluded women if the calculated interpregnancy interval (interval between two admissions minus the gestational age of second pregnancy) was less than four weeks and where gestational age at the second delivery was missing $(\mathrm{n}=60)$.

The primary end point was reproductive outcome in the second pregnancy-namely, miscarriage, ectopic pregnancy, termination, stillbirth, and live birth. Confounding factors comprised maternal age at delivery, socioeconomic status (classified according to the Carstairs index as "deprived" for categories 4 and above and "not deprived" for categories 1-3). ${ }^{17}$ As smoking status was unknown for $57.2 \%$ of the women, we carried out a sensitivity analysis including and excluding the smoking variable. The smoking variable is excluded in the results shown in this paper.

To assess the effects of interpregnancy interval on obstetric and perinatal complications we carried out a subgroup analysis of women who had a second pregnancy continuing beyond 24 weeks and resulting in a live birth. Secondary outcomes in ongoing pregnancies were pre-eclampsia, placenta praevia, placental abruption, mode of delivery, preterm and very preterm delivery, and low birthweight infants $(<2500 \mathrm{~g})$. We classified preterm birth as occurring at $36 \mathrm{com}-$ pleted weeks of gestation or less and very preterm birth at 32 weeks or less. All other outcomes listed in the database were defined using the ICD-9 classifications (international classification of diseases, ninth revision) until 1994 when the ICD-10 came into use. In addition to the sociodemographic variables, we adjusted for other pregnancy specific variables such as induction of labour, gestational age, and caesarean delivery, as appropriate.

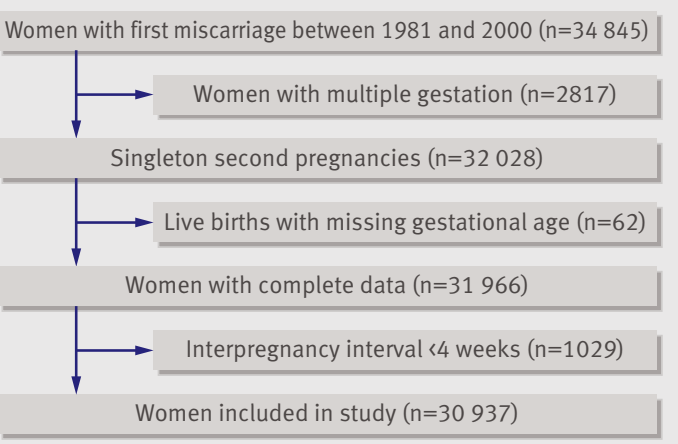

Flow of population selection 
Table 2 | Outcomes of second pregnancy after miscarriage in first pregnancy. Values are numbers (percentages)

\begin{tabular}{|c|c|c|c|c|c|}
\hline \multirow[b]{2}{*}{ Outcomes of second pregnancy } & \multicolumn{5}{|c|}{ Interpregnancy intervals (months) } \\
\hline & $<6(n=12744)$ & $6-12(n=7791)$ & $12-18(n=2958)$ & $18-24(n=1995)$ & $>24(\mathrm{n}=5449)$ \\
\hline Miscarriage & $1308(10.3)$ & 1004 (12.9) & $371(12.5)$ & $260(13.0)$ & $676(12.4)$ \\
\hline Ectopic pregnancy & $53(0.4)$ & $67(0.9)$ & $25(0.8)$ & $11(0.6)$ & $87(1.6)$ \\
\hline Termination & $297(2.3)$ & $367(4.7)$ & $168(5.7)$ & $141(7.1)$ & $537(9.9)$ \\
\hline Stillbirth & $70(0.5)$ & $55(0.7)$ & $26(0.9)$ & $13(0.7)$ & $35(0.6)$ \\
\hline Live birth & $10856(85.2)$ & $6154(79.0)$ & $2301(77.8)$ & $1530(76.7)$ & $3992(73.3)$ \\
\hline Other* & $160(1.3)$ & $144(1.8)$ & $67(2.3)$ & $40(2.0)$ & $122(2.2)$ \\
\hline
\end{tabular}

P<0.01.

*Includes molar pregnancy and pregnancy of unknown location.

\section{Statistical analysis}

To compare sociodemographic variables across the different bands of interpregnancy interval, we carried out a one way analysis of variance for normally distributed continuous variables, with $\chi^{2}$ tests for categorical variables. We used SPSS version 17 for the statistical analysis. Results are presented as both crude and adjusted odds ratios with $95 \%$ confidence intervals, using univariate and multivariate logistic regression (stepwise backward likelihood ratio method). In both the univariate and multivariate analyses we considered a P value of less than 0.05 to be significant. We used the Hosmer and Lemeshow test to assess goodness of fit of the models and the likelihood ratio test to assess the relative contribution of terms entered into the model. To assess interaction over time we entered into the model the interaction terms interpregnancy intervals $\times$ five year bands of year of first hospital admission.

A retrospective power calculation showed that the study had $99 \%$ power at the two sided $5 \%$ significance level to detect a difference of $2 \%$ or more in the occurrence of a second miscarriage, given that there were 12744 women in the exposed (interpregnancy interval $<6$ months) and 7791 women in the reference category (6-12 months).

\section{RESULTS}

Data were available on a total of 34845 women who had experienced a miscarriage in their first pregnancy and had a subsequent pregnancy. After the exclusion of multiple births and records with improbable interpregnancy intervals, 30937 women constituted the study population. The figure presents the flow of population selection.

Of these 30937 women, 12744 (41.2\%) conceived within six months of the miscarriage, 7791 (25.2\%) after 6-12 months, $2958(9.6 \%)$ after 12-18 months, $1995(6.4 \%)$ after 18-24 months, and $5449(17.6 \%)$ after 24 months.

Older women and those belonging to a higher social class tended to have shorter interpregnancy intervals (table 1). Compared with those with an interpregnancy interval of more than 24 months, women with an interpregnancy interval of less than six months were likely to be older (26.0 years (SD 5.4 years) v 23.9 (5.9) years; $\mathrm{P}<0.001)$ and from a less deprived Carstairs category at the time of their first pregnancy (4449 women, 34.9\% $v 1547$ women, $28.4 \% ; \mathrm{P}<0.001$ ), and less likely to have ever smoked (1261 women 9.9\% v 963 women, 17.7\% $\mathrm{P}<0.001)$.

Live birth rates were highest (10 856 women, 85.2\%) in women with an interpregnancy interval of less than six months and lowest $(\mathrm{n}=3992,73.3 \%)$ in those with an interpregnancy interval exceeding 24 months $(\mathrm{P}<0.01$; table 2). The data on termination of pregnancies also displayed a similar pattern, with longer interpregnancy intervals associated with a higher chance of termination in the next pregnancy.

After adjustment for maternal age at first pregnancy event, socioeconomic status, and year of first pregnancy, adverse pregnancy outcomes in women with an interpregnancy interval of less than six months differed significantly from the other groups (table 3 ). The models also included interaction terms: interpregnancy interval (six month bands) $\times$ year of first admission (five year bands). However, the interaction terms were found to be statistically significant only in the model for a second miscarriage (data available on request). Compared with the reference group (interpregnancy interval 6-12 months), women with an interpregnancy interval of less than six months were less likely to experience a miscarriage (adjusted odds ratio $0.66,95 \%$ confidence interval 0.57 to 0.77 ), termination $(0.43,0.33$ to 0.57$)$, or ectopic pregnancy $(0.48$, 0.34 to 0.69 ). The highest risk of adverse outcomes was in women with an interpregnancy interval of more than 24 months, who were more likely in their second pregnancy to have an ectopic pregnancy (1.97, 1.42 to 2.72$)$ or termination $(2.40,1.91$ to 3.01$)$.

Including smoking status (when available) as a covariate in a subset of women showed similar patterns of risk, although some odds ratios (such as that for ectopic pregnancy or stillbirth) were no longer significant as the confidence intervals were widened to include 1 or the models did not converge because of smaller numbers. (Data available on request.)

Table 4 shows the proportions of women with maternal and perinatal complications in their second pregnancy. Women with an interpregnancy interval of less than six months were less likely to have caesarean section, preterm delivery (before 36 weeks), or an infant of low birth weight $(<2500 \mathrm{~g})$ but were more likely to have an induced labour (table 5). Women with an interpregnancy interval of more than 24 months were most likely to have a preterm delivery $(1.21,1.07$ to 1.36$)$ or very preterm delivery $(1.40,1.11$ 
Table $3 \mid$ Crude and adjusted odds ratios of adverse pregnancy outcomes for different interpregnancy intervals

\begin{tabular}{|c|c|c|c|c|}
\hline \multirow[b]{2}{*}{ Second pregnancy outcomes } & \multicolumn{4}{|c|}{ Interpregnancy intervals (months) } \\
\hline & $<6$ & $12-18$ & $18-24$ & $>24$ \\
\hline \multicolumn{5}{|l|}{ Miscarriage: } \\
\hline Crude odds ratio $(95 \% \mathrm{Cl})$ & $0.77(0.71 \text { to } 0.84)^{\star}$ & $0.97(0.85$ to 1.10$)$ & 1.01 (0.88 to 1.17$)$ & 0.96 (0.86 to 1.06$)$ \\
\hline Adjusted† odds ratio $(95 \% \mathrm{Cl})$ & $0.66(0.57 \text { to } 0.77)^{\star}$ & $0.98(0.80$ to 1.21$)$ & 0.99 (0.78 to 1.27$)$ & $0.99(0.84 \text { to } 1.18)^{*}$ \\
\hline \multicolumn{5}{|l|}{ Ectopic pregnancy: } \\
\hline Crude odds ratio $(95 \% \mathrm{Cl})$ & $0.48(0.34 \text { to } 0.69)^{\star}$ & $0.99(0.62$ to 1.56$)$ & $0.64(0.34$ to 1.21$)$ & $1.87(1.36 \text { to } 2.58)^{*}$ \\
\hline Adjusted† odds ratio $(95 \% \mathrm{Cl})$ & $0.48(0.34 \text { to } 0.69)^{\star}$ & 0.99 (0.63 to 1.57$)$ & $0.65(0.34$ to 1.23$)$ & $1.97(1.42 \text { to } 2.72)^{*}$ \\
\hline \multicolumn{5}{|l|}{ Termination: } \\
\hline Crude odds ratio $(95 \% \mathrm{Cl})$ & $0.48(0.41 \text { to } 0.56)^{\star}$ & $1.22(1.01 \text { to } 1.47)^{\star}$ & $1.54(1.26 \text { to } 1.88)^{\star}$ & $2.21(1.93 \text { to } 2.54)^{\star}$ \\
\hline Adjusted† odds ratio $(95 \% \mathrm{Cl})$ & $0.43(0.33 \text { to } 0.57)^{\star}$ & 1.15 (0.83 to 1.59$)$ & $1.55(1.10 \text { to } 2.19)^{\star}$ & $2.40(1.91 \text { to } 3.01)^{\star}$ \\
\hline \multicolumn{5}{|l|}{ Stillbirth: } \\
\hline Crude odd ratio $(95 \% \mathrm{Cl})$ & $0.72(0.51$ to 1.03$)$ & 1.26 (0.79 to 2.02$)$ & $0.95(0.52$ to 1.75$)$ & 0.98 (0.64 to 1.50$)$ \\
\hline Adjusted† odds ratio $(95 \% \mathrm{Cl})$ & $0.70(0.38$ to 1.29$)$ & $1.20(0.54$ to 2.67$)$ & $0.83(0.28$ to 2.44$)$ & 1.07 (0.54 to 2.15$)$ \\
\hline \multicolumn{5}{|c|}{$\begin{array}{l}\text { Reference category was interpregnancy interval of } 6-12 \text { months. Interaction terms interpregnancy interval } \times \text { year of admission in five year bands were } \\
\text { entered into the model, but only that for miscarriage was statistically significant. } \\
{ }^{*} \times 0.05 \text {. } \\
\text { †Adjusted for maternal age at first pregnancy, Carstairs social deprivation category, and year of first pregnancy. }\end{array}$} \\
\hline
\end{tabular}

to1.70) than women with an interpregnancy interval of 6-12 months. They were also more likely to have a caesarean section $(1.39,1.26$ to 1.54$)$ or an infant of low birth weight $(1.23,1.02$ to 1.48$)$.

No association was found between interpregnancy interval after miscarriage and pre-eclampsia, placenta praevia, or placental abruption in the second ongoing pregnancy.

\section{DISCUSSION}

Women who conceived within six months of an initial recorded miscarriage had the lowest rates of miscarriage, ectopic pregnancy, and termination in a subsequent pregnancy than those who conceived later. They were also least likely to deliver preterm or very preterm or have an infant of low birth weight but were at highest risk of induced labour. Women with an interpregnancy interval of more than 24 months had the highest risks of ectopic pregnancy and termination and were most likely to have a caesarean section or preterm delivery and an infant of low birth weight.

\section{Comparison with other studies}

The only studies to have dealt with the effect of interpregnancy intervals on pregnancy outcome after miscarriage have been from developing countries with different systems of family planning, access to maternity care, and methods of data collection. In the developing world, women also tend to bear children at a younger age, thus reducing their age dependent risk of an adverse pregnancy outcome.

Additionally, most published research has focused on interpregnancy intervals after live birth. Few have investigated interpregnancy intervals after other types of pregnancy outcomes, possibly because of smaller numbers of women in these groups. One study reported perinatal outcomes after varying interpregnancy intervals and according to the type of pregnancy outcome that began the interval, ${ }^{18}$ but data in this study were drawn from a demographic surveillance site in Bangladesh where induced abortion is illegal, possibly resulting in over-reporting of miscarriages owing to the sensitivity of this information. Moreover, the reference group was women who had an initial live birth, thus potentially inflating the risk of adverse outcomes after miscarriage.

Another study examined the effects of varying interpregnancy intervals after pregnancy termination (both miscarriage and induced) on maternal and perinatal health in a sample of 258108 Latin American women. An interpregnancy interval of less than six months after abortion was independently associated with increased risks of adverse maternal and perinatal outcomes, including maternal anaemia, low birthweight infants, and preterm delivery in the next pregnancy. ${ }^{19}$ This study did not, however, differentiate between induced abortion and miscarriage in the index pregnancy. This limitation, along with the geographical context of the study, may affect the generalisability of the results to other regions. Despite these limitations, these data were the sole basis for a WHO recommendation ${ }^{9}$ on birth spacing after abortion, calling for more studies on pregnancy intervals after miscarriage in different populations and healthcare settings.

Previous Scottish studies ${ }^{20}$ have shown that a short interpregnancy interval after stillbirth is associated with increased risks in the next pregnancy but did not explore the association between interpregnancy interval and outcomes after miscarriage. Two other European reports ${ }^{2122}$ have suggested that adverse pregnancy outcomes are more likely to occur after miscarriage in women with increasing interpregnancy intervals.

Much of the existing literature comprises studies on perinatal outcomes after a previous live birth and suggests that short interpregnancy intervals are detrimental to maternal and perinatal wellbeing. Our results challenge this view but are consistent with the maternal depletion hypothesis suggested by one study. ${ }^{23}$ The researchers propose that decreasing levels of folate in 
Table $4 \mid$ Maternal and perinatal complications by interpregnancy interval in women with live births in pregnancy after initial miscarriage. Values are numbers (percentages) of women

\begin{tabular}{|c|c|c|c|c|c|c|}
\hline \multirow{2}{*}{$\begin{array}{l}\text { Complications in second ongoing } \\
\text { pregnancy }\end{array}$} & \multicolumn{5}{|c|}{ Interpregnancy interval (months) } & \multirow[b]{2}{*}{$P$ value } \\
\hline & $<6(n=10856)$ & $6-12(n=6154)$ & $12-18(n=2301)$ & $18-24(n=1530)$ & $224(n=3992)$ & \\
\hline Pre-eclampsia & $485(4.5)$ & $265(4.3)$ & $117(5.1)$ & $72(4.7)$ & $180(4.5)$ & 0.637 \\
\hline Placenta praevia & $86(0.8)$ & $57(0.9)$ & $23(1.0)$ & $20(1.3)$ & $38(1.0)$ & 0.323 \\
\hline Placental abruption & $417(3.8)$ & $269(4.4)$ & $106(4.6)$ & $74(4.8)$ & $187(4.8)$ & 0.073 \\
\hline Induction of labour & $2963(27.3)$ & $1599(26.0)$ & $617(26.8)$ & $454(29.7)$ & $1065(26.7)$ & 0.050 \\
\hline Caesarean delivery & 2002 (18.5) & $1230(20.0)$ & $485(21.0)$ & $301(19.7)$ & $939(23.5)$ & $<0.01$ \\
\hline Preterm delivery ( 336 weeks) & $782(7.2)$ & $500(8.1)$ & $196(8.5)$ & $125(8.2)$ & $422(10.6)$ & $<0.01$ \\
\hline Very preterm delivery ( $\$ 32$ weeks) & $182(1.7)$ & $117(1.9)$ & $55(2.4)$ & $34(2.2)$ & $122(3.1)$ & $<0.01$ \\
\hline Low birth weight (<2500 g) & $776(7.15)$ & $527(8.6)$ & 227 (9.9) & $131(8.6)$ & $473(11.9)$ & $<0.01$ \\
\hline
\end{tabular}

the mother after the fifth month of gestation onwards that remain low for an extended period of time after delivery could result in folate insufficiency in women with short interpregnancy intervals leading to neural tube defects, intrauterine growth restriction, and preterm birth. Additionally, breast feeding after a live birth leads to further depletion of folate in the mother, ${ }^{18}$ compounding this effect. As most miscarriages occur in the first trimester and are not associated with breast feeding, women conceiving again soon after their pregnancy loss may not have any depletion of vital nutrients and thus have a reduced risk of adverse outcomes.

Some authors attribute the higher risks of adverse maternal outcomes after a short interpregnancy interval to socioeconomic status, unstable lifestyles, failure to use healthcare services, unplanned pregnancies, and other behavioural or psychological factors. ${ }^{24}$ Our data, however, show the effect of interpregnancy interval after a miscarriage to be different. In our cohort, fewer women with interpregnancy intervals of less than six months were classed as deprived compared with any of the other groups $(65.1 \% \quad v \quad 71.6 \%$ in women with an interpregnancy interval $>24$ months). Thus, although a short interpregnancy interval after a live birth is more likely in a woman with poor education and with limited access to family planning resources, this may not be the case after a miscarriage, at least in our population of women. It is unknown why a longer interval is associated with adverse outcomes such as miscarriage or ectopic pregnancy. One study offered two hypotheses. ${ }^{11}$ Firstly, a pregnancy may enhance the functional capacity of the reproductive system, which can wane over time, and with longer intervals the risks to a mother and baby may resemble those in a primigravida. An alternative hypothesis is that factors associated with underlying subfertility, which can increase the time to the next pregnancy, could result in adverse perinatal outcomes. ${ }^{24}$

It is also possible, as suggested by a previous study, ${ }^{6}$ that women who become pregnant soon after an initial miscarriage may have been highly motivated such that their health related behaviours may have favoured more successful pregnancies. Of the studies that distinguished between the different types of initial pregnancies, one ${ }^{18}$ also found that the rates of live birth in the subsequent pregnancy decreased in a linear fashion with increasing interpregnancy intervals.

In a systematic review ${ }^{25}$ it was reported that women who conceived again within a short time after delivery tended to have a lower parity than those who waited longer. A higher proportion of women with an interpregnancy interval of less than six months also did not have antenatal care; again, this could have influenced the outcomes reported in their review. Previous studies have reported that inadequate use of prenatal care is a more potent predictor of adverse pregnancy outcomes than interpregnancy interval. ${ }^{2627}$ We were unable to estimate the potential confounding effects of quality of, access to, and use of prenatal care in our population. Nevertheless, as medical care is available to all, free of charge at the point of access in Scotland, this is unlikely to be a major limitation in our study.

\section{Strengths and weaknesses of the study}

The main strength of this research lies in its large population based nature, and the use of routinely collected data on hospital admissions. The data are cross checked with other hospital data for completeness and accuracy. A set of validation rules is applied to all Scottish morbidity records both locally, before submission to Information Services Division, and again once each record has been submitted. These checks can detect errors where information is missing, invalid, or does not conform to a logical sequence of events. The data monitoring team also carry out local quality checks by investigating anomalies in Scottish morbidity records and ensuring that data are amended through usual submission protocols. Regular cleanup programs on national files are undertaken. Additionally, the data quality assurance team routinely compare samples of data from the Scottish national database with the original patient case notes to ensure correct interpretation of the data definitions and recording rules.

This study is not without its limitations. One of the problems in using routinely collected data is the potential lack of uniformity in the documentation of gestational age and outcomes of interest as well as the possibility of misclassification. As parity is not routinely recorded in the morbidity records for women who have early pregnancy loss, we were unable to 
Table $5 \mid$ Crude and adjusted odds ratios for complications in second pregnancy after initial miscarriage

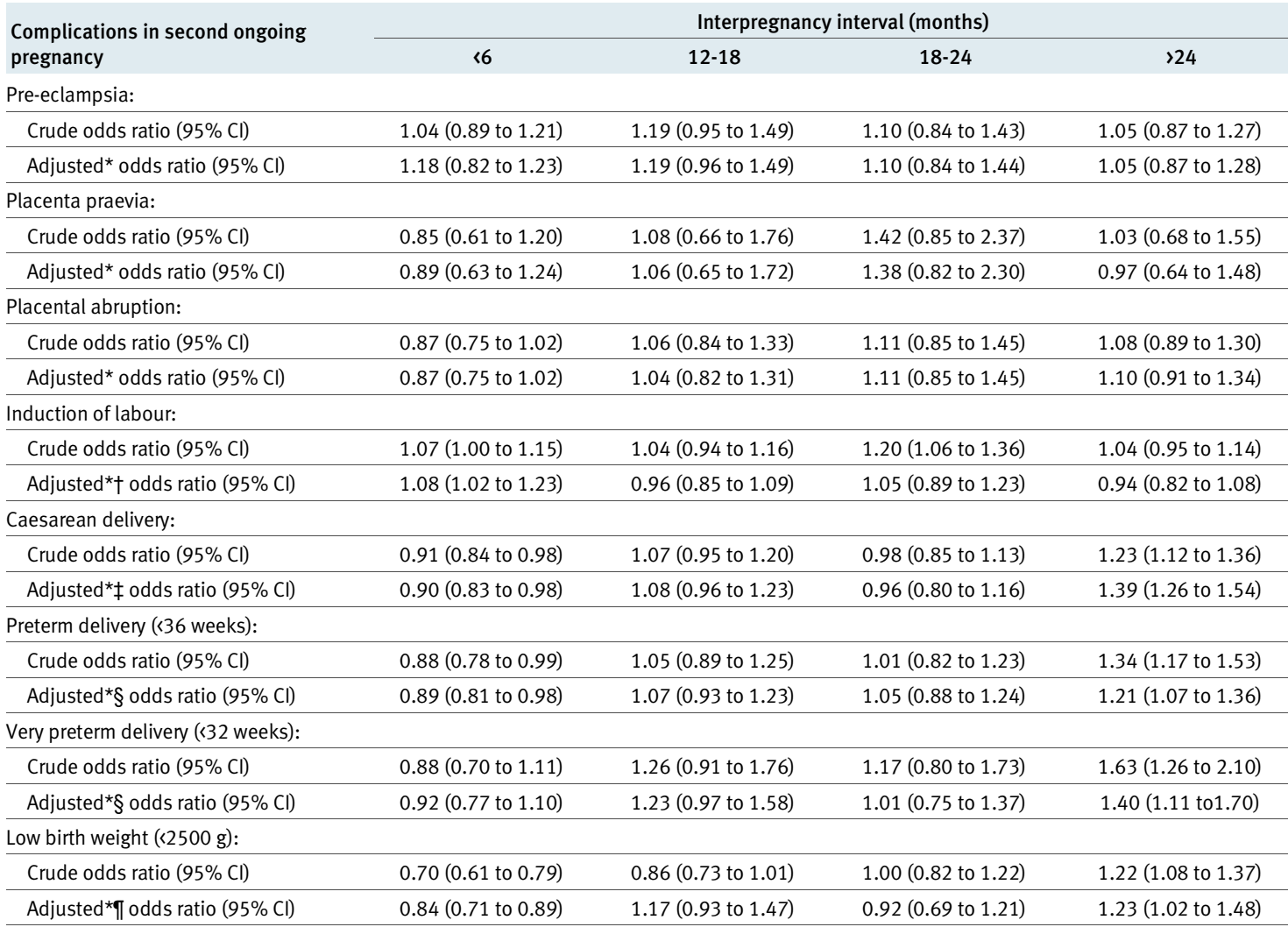

Reference category was interpregnancy interval of 6-12 months.

*Adjusted for maternal age at first pregnancy, Carstairs social deprivation category, and year of first miscarriage.

†Also adjusted for pre-eclampsia and placental abruption.

$\ddagger$ Also adjusted for pre-eclampsia, placenta praevia, and placental abruption.

$\S$ Also adjusted for pre-eclampsia, placenta praevia, placental abruption, and induction of labour.

TAlso adjusted for gestation at delivery.

confirm if the earliest miscarriages recorded were indeed the first pregnancy event. For women who went on to have a delivery, it was possible to check if an initial miscarriage was recorded. However, we believe that the large size of the dataset may offset any minor discrepancies such that the overall pattern of any trends remains unaffected. Further disadvantages in using information from the Scottish dataset include potential inaccuracies in estimation of times between pregnancies due to misclassification of hospital admissions that could have been the result of the same event. This study also spans a long period during which advances in obstetric practice may have influenced the outcomes of interest. Specifically, the routine use of ultrasound for diagnosis, increasing availability of assisted reproduction techniques, and the formation of dedicated early pregnancy units for the management of pregnancy loss all represent recent advances and are likely to affect outcomes. However, as the women in all the interpregnancy interval groups were identified from the same period, and the year of occurrence of the first pregnancy event was included as a covariate in the model, this is unlikely to have produced a significant effect on the results. We also looked for interaction of interpregnancy intervals over time, but this was only found to be statistically significant in some women who had a second miscarriage.

Additionally, this study examined miscarriages that led to hospital contact only, and results therefore cannot be generalised to all women with a miscarriage. However, most women in Scotland who are aware that they have miscarried are likely to attend hospitals. Moreover, there is a possibility that adverse second pregnancy outcomes may be over-represented in our sample as women who were pregnant for the first time towards the latter part of the study period may have had a second pregnancy of normal duration. These women will have been missed as they had no reason to have any hospital admissions at that time. The causes of miscarriages in the second trimester are different from those in the first, which are more common and can be missed. We therefore did a subgroup analysis on women with an initial second trimester miscarriage (data not shown) and found that the results were consistent with the findings of this study. Unrecorded miscarriages could also result in misclassification bias in our estimates of interpregnancy intervals. ${ }^{13} \mathrm{We}$ are also uncertain whether the intervals between pregnancies were voluntary or not. Women taking longer to conceive may be more likely to have worse pregnancy 


\section{WHAT IS ALREADY KNOWN ON THIS TOPIC}

Little is known about the effect of the intervals between pregnancies after an initial miscarriage

Previous studies have focused on the interpregnancy interval after a live birth or focused on women in developing countries

\section{WHAT THIS STUDY ADDS}

After an initial miscarriage, women from a developed country had the best outcomes in a subsequent pregnancy if they conceived again within six months

outcomes owing to factors causing a delay in fertility rather than the interpregnancy interval itself.

It should also be mentioned that the Scottish population is a relatively homogeneous, stable population. Although this may help reveal differences that may not surface in a heterogeneous society, it means that the results of this study may not be generalised to other populations. Finally, the potential for residual confounding by factors unknown exists; a substantial amount of data on the women's smoking status was missing and other potentially important confounding factors (for example, body mass index) were not measured.

\section{Implications for research}

Sporadic miscarriage is common and given the divided nature of the literature it is clear that further research needs to be done on the influence of interpregnancy intervals on pregnancy outcome after a miscarriage in different sociocultural settings. The ability to adjust for additional confounding factors such as ethnicity, change of partner, maternal weight, and separation of voluntary from involuntary interpregnancy intervals would lend credibility to future research.

\section{Implications for clinical practice}

Our research shows that it is unnecessary for women to delay conception after a miscarriage. As such the current WHO guidelines may need to be reconsidered. In accordance with our results, women wanting to become pregnant soon after a miscarriage should not be discouraged. There may be cases where a delay is desirable, for example if there are signs of infection, and women should be advised appropriately. Also, in a molar pregnancy, current guidelines advise delaying the next pregnancy for at least one year after treatment. ${ }^{28}$

After a miscarriage, women should also be counselled about how to optimise their own health before and during pregnancy in preparation for subsequent conception. The potential negative effects of delaying the next pregnancy for more than 18-24 months should also be discussed with couples, allowing them to make a fully informed decision about future pregnancy.

\section{Conclusion}

In the Scottish population, women who conceive within six months of an initial miscarriage have the best outcomes and lowest complication rates in their subsequent pregnancy.

We thank Joanne Hatty for extraction of data from the Scottish morbidity records databases and jill Mollison for statistical advice on the revised version of this paper.

Contributors: ERL analysed the data and wrote the first draft of the paper. SohB extracted data and supervised the data analysis and writing of the first draft of the paper. SB conceived the study and was responsible for overall supervision. NCS helped with the clinical interpretation of the results and commented on the first draft of the paper. All authors contributed to the writing of the final draft of the paper. SohB is the guarantor.

Funding: This research was partially funded by the Chief Scientist's Office in Scotland (project No CZG_2_283). SB and SohB were employed by the University of Aberdeen at the time of doing this research and are independent from the funders. ERL is a medical student and NCS is employed by NHS Grampian. The findings and their interpretation in this study are the authors' own, and neither the funders nor the sponsors played any part in arriving at the conclusions.

Competing interests: All authors have completed the unified competing interest form (available on request from the corresponding author) and declare that: ERL,SB, NCS, and SohB have support from the University of Aberdeen for the submitted work; ERL, SB, NCS, and SohB have no relationships with companies that might have an interest in the submitted work in the previous three years; and ERL, SB, NCS, and SohB have no non-financial interests that may be relevant to the submitted work. Ethical approval: This research proposal was approved by the privacy advisory committee of the Information and Services Division N HS Scotland. Formal ethical approval was not considered necessary by North of Scotland Research Ethics Service, as only anonymised data were analysed in this study.

Data sharing: No additional data available.

1 Wilcox AJ, Weinberg CR, O'Connor JF, Baird DD, Schlatterer JP, Canfield RE. Incidence of early loss of pregnancy. N Engl J Med 1988;319:89-94.

2 Hathout H, Kasrawi R, Moussa MA, Saleh AK. Influence of pregnancy outcome on subsequent pregnancy. Int J Gynaecol Obstet 1982;20:145-7.

3 Bhattacharya S, Townend J, Shetty A, Campbell D, Bhattacharya S. Does miscarriage in an initial pregnancy lead to adverse obstetric and perinatal outcomes in the next continuing pregnancy? BJOG 2008;115:1623-9.

4 Goldstein RR, Pruyn MA, Croughan MS, Robertson PA. Neonatal outcomes in immediate versus delayed conceptions after spontaneous abortion: a retrospective case series. Am J Obstet Gynecol 2002;186:1230-6.

5 Wyss P, Biedermann K, Huch A. Relevance of the miscarriage-new pregnancy interval. J Perinat Med 1994;22:235-41.

6 Rud B, Klunder K. The course of pregnancy following spontaneous abortion. Acta Obstet Gynecol Scand 1985;64:277-8.

7 Cuisinier M, Janssen H, De Graauw C, Bakker S, Hoogduin C. Pregnancy following miscarriage: course of grief and some determining factors. I Psychosom Obstet Gynecol 1996;17:168-74.

8 Franche R, Bulow C. The impact of a subsequent pregnancy on grie and emotional adjustment following a perinatal loss. Infant Ment Health / 1999;20:175-87.

9 World Health Organization. Report of a WHO technical consultation on birth spacing. 2005. www.who.int/making_pregnancy_safer/ documents/birth spacing.pdf.

10 Zhu BP, Le T. Effect of interpregnancy interval on infant low birth weight: a retrospective cohort study using the Michigan Maternally Linked Birth Database. Matern Child Health J 2003;7:169-78.

11 Zhu BP, Rolfs RT, Nangle BE, Horan JM. Effect of the interval between pregnancies on perinatal outcomes. N Engl J Med 1999;340:589-94.

12 Conde-Agudelo A, Belizan JM. Maternal morbidity and mortality associated with interpregnancy interval: cross sectional study. $B M$ J 2000;321:1255-9.

13 Fuentes-Afflick E, Hessol NA. Interpregnancy interval and the risk of premature infants. Obstet Gynecol 2000;95:383-90.

14 Aref-Adib M, Freeman-Wang T, Ataullah I. The older obstetric patient. Obstetr Gynaecol Reprod Med 2008;18:43-8.

15 Nabukera SK, Wingate MS, Salihu HM, Owen J, Swaminathan S, Alexander GR, et al. Pregnancy spacing among women delaying initiation of childbearing. Arch Gynecol Obstet 2009;279:677-84.

16 Cole SK. Scottish maternity and neonatal records. In: Chalmers I, Mcllwaine GM, eds. Perinatal audit and surveillance. Royal College of Obstetricians and Gynaecologists, 1980:39-51.

17 Morris R, Carstairs V. Which deprivation? A comparison of selected deprivation indexes. J Public Health 1991;13:318-26. 
18 DaVanzo J, Hale L, Razzaque A, Rahman M. Effects of interpregnancy interval and outcome of the preceding pregnancy on pregnancy outcomes in Matlab, Bangladesh. BJOG 2007;114:1079-87.

19 Conde-Agudelo A, Belizan JM, Breman R, Brockman SC, Rosas-Bermudez A. Effect of the interpregnancy interval after an abortion on maternal and perinatal health in Latin America. Int Gynaecol Obstet 2005;89:34-40S.

20 Smith GC, Pell JP, Dobbie R. Interpregnancy interval and risk of preterm birth and neonatal death: retrospective cohort study. BMJ 2003;327:313.

21 Basso 0, Olsen J, Christensen K. Risk of preterm delivery, low birthweight and growth retardation following spontaneous abortion: a registry-based study in Denmark. Int J Epidemiol 1998;27:642-6.

22 Hassan MA, Killick SR. Is previous aberrant reproductive outcome predictive of subsequently reduced fecundity? Hum Reprod 2005;20:657-64.
23 Smits L, Essed G. Short interpregnancy intervals and unfavourable pregnancy outcome: role of folate depletion. Lancet 2001;358:2074-7.

24 Thomson F, Shanbhag S, Templeton A, Bhattacharya S. Obstetric outcome in women with subfertility. BJOG 2005;112:632-7.

25 Conde-Agudelo A, Rosas-Bermudez A, Kafury-Goeta A. Effects of birth spacing on maternal health: a systematic review. Am J Obstet Gynecol 2007;196:297-308.

26 Miller JE. Birth intervals and perinatal health: an investigation of three hypotheses. Fam Plann Perspect 1991;23:62-70.

27 Rawlings JS, Rawlings VB, Read JA. Prevalence of low birth weight and preterm delivery in relation to the interval between pregnancies among white and black women. N Engl J Med 1995;332:69-74.

28 Royal College of Obstetricians and Gynaecologists. The management of gestational trophoblastic disease. RCOG, 2010.

Accepted: 24 June 2010 